\title{
Enhanced absorption and electrical modulation of graphene based on the parity-time symmetry optical structure
}

\author{
Lingjun Yi (易凌俊) and Changhong Li (李长红) \\ School of Electronic Information, Qingdao University, Qingdao 266071, China \\ *Corresponding author: jiluch@126.com \\ Received September 26, 2021 | Accepted November 10, 2021 | Posted Online November 25, 2021
}

\begin{abstract}
In order to realize the ultrastrong absorption of graphene with electrical modulation properties, we designed a composite structure of graphene and parity-time (PT) symmetry photonic crystal, which is achieved by placing the graphene layer on the top layer of the PT symmetry photonic crystal. In this paper, the absorption properties of graphene and the electrical modulating properties of the structure were theoretically analyzed based on the transfer matrix method. The result shows that the proposed structure can achieve the absorption of $31.5 \mathrm{~dB}$ for the communication wavelength of $1550 \mathrm{~nm}$; meanwhile, by setting the electric field intensity to $\pm 0.02 \mathrm{~V} / \mathrm{nm}$, the absorption of graphene can be largely modulated to realize an electrically switchable effect, the modulation depth of graphene absorption can reach nearly $100 \%$, and the operation speed is also close to $8.171 \mathrm{GHz}$. This investigation provides a novel approach to design graphene-based optoelectronic devices and optical communication devices.
\end{abstract}

Keywords: parity-time symmetry; ultrastrong absorption; electrical modulation; graphene; transfer matrix method. DOI: 10.3788/COL202220.022201

\section{Introduction}

Graphene is a two-dimensional (2D) atomic scale hexagonal carbon isomorphism. Its conduction band and valence band meet at the Dirac point. Accordingly, graphene is equivalent to a zero-gap semiconductor with electron mobility exceeding $15,000 \mathrm{~cm}^{2} \cdot \mathrm{V}^{-1} \cdot \mathrm{s}^{-1}$ at room temperature ${ }^{[1]}$, which endows graphene excellent electrical and optical properties and prompts a wide range of potential applications in the perfect absorption of optoelectronic devices, optical communication systems, as well as sensing devices ${ }^{[2-8]}$. Due to monolayer graphene itself being incapable of exciting surface plasmon resonances, its theoretical absorption efficiency in air is exclusively about $2.3 \%$ of the single-pass light ${ }^{[9]}$, which seriously obstructs its application range and device performance. Thus, researchers have proposed a variety of structures like multilayer thin film photonic crystal by using the photonic local state formed between graphene and photonic crystals or based on surface plasmon resonance to improve the absorption of graphene. For instance, Zhu et al. proposed a composite structure of graphene and one-dimensional photonic crystals (1D-PCs), where graphene absorption can reach $0.88(-0.555 \mathrm{~dB})^{[10]}$. Geng et al. presented a patterned graphene-hexagonal boron nitride $(\mathrm{hBN})$ metasurface, where the absorption of graphene in the structure is close to one (nearly $0 \mathrm{~dB})^{[11]}$. By exciting Dirac plasmons on nanopatterned monolayer graphene, Safaei et al. increased the absorption of graphene to $90 \%(-0.458 \mathrm{~dB})^{[12]}$. However, the absorption efficiency only increases by one or two orders of magnitude in these literatures, and the absorption efficiency of graphene needs to be further enhanced for optoelectronic detection and optical communication applications.

For the general periodic photonic crystal structure, when studying the transmission, reflection, and absorption of light, the gain and loss of the medium are often not considered, or the loss of the medium is regarded as an adverse factor. But, for optical structures that satisfy parity-time (PT) symmetry, quantum dots are anthropogenically introduced into the substrate medium forming gain and loss dielectric layers. When the gain and loss dielectric layers are accurately anastomotic, a bizarre optical transmission phenomenon can be produced under the action of external pump energy ${ }^{[13-15]}$, and therefore the PT symmetry optical microcavity structure could be also an excellent option used to heighten the absorption of graphene.

Based on the special optical properties of the PT symmetry structure, we take the graphene as the top layer, with the photonic crystals satisfying the PT symmetry structure as the bottom layer, so we realized the ultrastrong absorption of 
graphene. Meanwhile, the modulation of graphene absorption is achieved by using the photoelectric effect of $\mathrm{LiNbO}_{3}$ crystals.

\section{Model Design and Theoretical Calculation}

The overall structure is designed as shown schematically in Fig. 1, where each dielectric layer can be simply represented as $\mathrm{G}(\mathrm{ADB})^{N} \mathrm{C}(\mathrm{BDA})^{N}$. Thereinto, the $\mathrm{G}$ deputies graphene layer is placed to the forward side of the PT symmetry photonic crystal. For the underlying structure, the gain-loss layers are formed by doping quantum dots in the substrate dielectric silica, denoted by A and B, respectively. Such gain or loss dielectric layers can be realistically manufactured by analogy to quantum well amplifiers or photorefractive structures. We can use a modified solution doping method in the modified chemical vapor deposition (MCVD) process to develop a gain-doped or lossdoped silica substrate ${ }^{[16]}$. The refractive index of the A or B layer can be quantitatively described by the Lorentz model ${ }^{[17]}$ :

$$
n_{\mathrm{A} / \mathrm{B}}=\left(\varepsilon_{\mathrm{SiO}_{2}}+\frac{\alpha \omega_{0}^{2}}{\omega_{0}^{2}-\omega^{2}-\omega \gamma_{1} i}\right)^{\frac{1}{2}},
$$

where $\gamma_{1}=2.5 \times 10^{14} \mathrm{~s}^{-1}$ is the damping coefficient; $\omega_{0}=$ $1.216 \times 10^{15} \mathrm{~s}^{-1}$ is the resonance angular frequency, which corresponds to the resonance wavelength of $1550 \mathrm{~nm}$; $\omega$ indicates the incident angle frequency; $\alpha$ is the macroscopic Lorentz oscillation intensity, which characterizes the relationship among the system gain, the concentration of doping quantum dots, and the distribution of excited state quantum dots. When the whole structure satisfies the PT symmetry condition, the absolute value of the macroscopic Lorentz oscillation intensity of layer A is tantamount to layer $\mathrm{B}$, and the value of $\alpha$ will have an effect on the absorption efficiency of graphene, which will be demonstrated in the third part. $\varepsilon_{\mathrm{SiO}_{2}}$ denotes the dielectric constant of the basal silica, which can be described by the Sellmeier dispersion relation as an approximation ${ }^{[18]}$ :

$$
\begin{aligned}
\varepsilon_{\mathrm{SiO}_{2}}= & 1+\frac{0.7 \lambda}{\lambda^{2}-6.8 \times 10^{-8}}+\frac{0.41 \lambda}{\lambda^{2}-1.16 \times 10^{-7}} \\
& +\frac{0.9 \lambda}{\lambda^{2}-9.896 \times 10^{-6}} .
\end{aligned}
$$

The $\mathrm{D}$ and $\mathrm{C}$ layers represent $\mathrm{LiNbO}_{3}$ crystal, which is a typical optical functional material, and its refractive index will vary

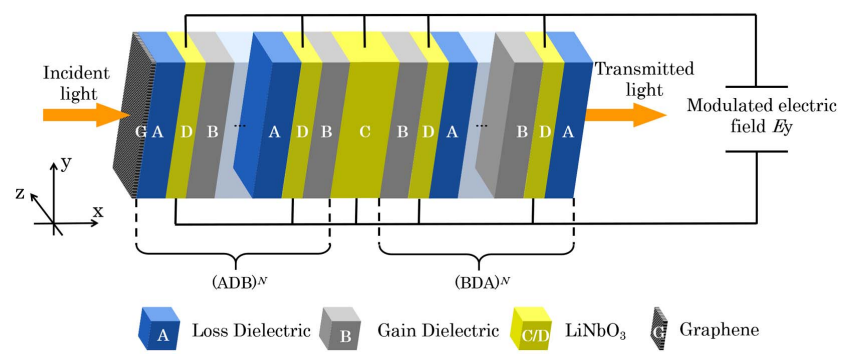

Fig. 1. Schematic diagram of the proposed structure. with the applied electric field and satisfies the ellipsoid equation. In this paper, the TE wave is selected as the incident wave, and its electric field is distributed along the $z$ axis. When the applied electric field direction is along the $y$ axis, the refractive index of the D or C layer can be expressed as ${ }^{[19]}$

$$
n_{\mathrm{D} / \mathrm{C}}=n_{0}-\frac{1}{2} n_{0}^{3} \gamma_{2} E_{y}
$$

where $n_{0}=2.286, \gamma_{2}=8.6 \times 10^{-3} \mathrm{~nm} / \mathrm{V}$ is an electro-optic constant, and $E_{y}$ represents the external electric field intensity along the $y$ axis. When implementing the electric-optical modulation of graphene absorption, the electro-optic control principle of the resonator shown in literature ${ }^{[20]}$ can be used to apply a modulating voltage between two metal parallel electrodes of the structure shown in Fig. 1 by accurately matching the inter-stage distance, inter-stage voltage, and dielectric constant, and then the modulating electric field intensity $E_{y}$ can be obtained.

For the graphene layer on the top, the optical properties are closely related to its complex surface conductivity $\sigma_{\mathrm{G}}$. Its effective dielectric constant can be expressed as ${ }^{[21]}$

$$
\varepsilon_{\mathrm{G}}=1+\frac{\mathrm{i} \sigma_{\mathrm{G}}}{\omega \varepsilon_{0} d_{\mathrm{G}}}
$$

where $\varepsilon_{0}$ is the vacuum dielectric constant, $d_{\mathrm{G}}=0.34 \mathrm{~nm}$ is the thickness of monolayer graphene, $\omega$ is the angular frequency of incident light, and $\sigma_{\mathrm{G}}$ is the surface conductivity of graphene under random-phase approximation, which can be expressed as

$$
\sigma_{\mathrm{G}}=\sigma_{\text {in }}+\sigma_{\text {out }} .
$$

Herein,

$$
\begin{gathered}
\sigma_{\text {in }}=\frac{i \mathrm{e}^{2}}{4 \pi \hbar} \ln \left|\frac{2 \mu-\hbar(\omega+i / \tau)}{2 \mu+\hbar(\omega+i / \tau)}\right| \\
\sigma_{\text {out }}=\frac{i e^{2} k_{B} T}{\pi \hbar^{2}(\omega+i / \tau)}\left\{\frac{\mu}{k_{B} T}+2 \ln \left[\exp \left(-\frac{\mu}{k_{B} T}\right)+1\right]\right\},
\end{gathered}
$$

where $\hbar=h / 2 \pi$ is the reduced Planck constant $(h=6.6260 \times$ $\left.10^{-34} \mathrm{~J} \cdot \mathrm{s}\right), e$ is electron charge, $k_{B}$ is the Boltzmann constant, $T=300 \mathrm{~K}$ is the Kelvin temperature, $\mu$ is the chemical potential, which is determined by the electron concentration and can be controlled by the gate voltage ${ }^{[22]}$, and $\tau=0.5 \times 10^{-14} \mathrm{~s}$ denotes the relaxation time of the graphene layer.

The transmission matrix method (TMM) is used to study the absorption characteristics of graphene in this paper, and the detailed description of the TMM is shown in relevant literature $^{[23]}$. It should be emphasized that when the TMM is used for simulation calculation in this paper, the graphene monolayer on the top layer is regarded as a finite-width layer with a thickness of $d_{\mathrm{G}}=0.34 \mathrm{~nm}$. Accordingly, the absorption of the graphene layer can be described by the following two processes: firstly, when incident light enters the underlying PT symmetry photonic crystal structure through the graphene layer, the initial 
absorption of incident light is caused by graphene; and, secondly, the Bragg reflection of incident light is produced by the bottom layer PT symmetry photonic crystal structure, when the reflected light passes through the graphene layer. We can calculate the absorption in the graphene layer by Poynting vectors $^{[24]}$ :

$$
A_{\mathrm{G}}=\frac{\left(\mathbf{S}_{\text {total }}^{+}-\mathbf{S}_{\text {total }}^{-}\right)-\left(\mathbf{S}_{\mathrm{PT}}^{+}-\mathbf{S}_{\mathrm{PT}}^{-}\right)}{\mathbf{S}_{\text {total }}^{+}},
$$

where $\mathbf{S}_{\text {total }}^{+}$and $\mathbf{S}_{\text {total }}^{-}$represent the total incident and reflected Poynting vectors of the whole structure, respectively; $\mathbf{S}_{\mathrm{PT}}^{+}$and $\mathrm{S}_{\mathrm{PT}}^{-}$represent the incident and reflected Poynting vectors of the PT symmetry 1D-PC, respectively. According to the total reflection coefficient and transmission coefficient of the whole structure, the absorption of graphene can be derived by Poynting vector expression:

$$
A_{\mathrm{G}}=1-r_{\mathrm{total}}^{2}-\frac{t_{\mathrm{total}}^{2}}{t_{\mathrm{PT}}^{2}}\left(1-r_{\mathrm{PT}}^{2}\right),
$$

in which $r_{\text {total }}$ and $r_{\mathrm{PT}}$ represent the reflection coefficients of the holistic structure and the underlying PT symmetry structure, respectively; $t_{\text {total }}$ and $t_{\mathrm{PT}}$ represent the transmission coefficients of the overall structure and the underlying PT symmetry structure, respectively.

\section{Results and Discussion}

To satisfy the PT symmetry condition and make the defect mode be generated at $1550 \mathrm{~nm}$, in numerical calculations, the thickness of the A and B layers is set to $d_{\mathrm{A}}=d_{\mathrm{B}}=536.85 \mathrm{~nm}$ for the underlying PT symmetry photonic crystal. The macroscopic Lorentz oscillation intensity $\alpha_{\mathrm{A}}=2.0 \times 10^{-4}$ and $\alpha_{\mathrm{B}}=-2.0 \times$ $10^{-4}$. By adjusting the thickness of the $\mathrm{D}$ dielectric layer to $d_{\mathrm{D}}=449 \mathrm{~nm}$, the thickness of the C dielectric layer is $d_{\mathrm{C}}=2 d_{\mathrm{D}}$, as the defect layer of the whole structure. Without the external electric field, the refractive index of the $\mathrm{D}$ and $\mathrm{C}$ layers is $n_{0}=2.286$. The chemical potential $\mu=0.3 \mathrm{eV}$ for monolayer graphene, and the PT symmetry structure period is $N=6$. Figure 2 shows the transmission and reflection of the whole structure and the absorption spectra of monolayer graphene with the condition of normal incidence. Since there is a large difference in the numerical value, here, logarithmic transformation, with decibels $(\mathrm{dB})$ as the unit, is taken for the transmission during calculation. The transformation relation can be written as $Y(\mathrm{~dB})=10 \lg Y$, where $Y$ can represent the transmission and reflection of the whole structure and the absorption of monolayer graphene.

As clearly seen in Fig. 2, at the wavelength of $1550 \mathrm{~nm}$, the transmission and reflection of the whole structure can reach $13.71 \mathrm{~dB}$ and $11.72 \mathrm{~dB}$, respectively. The absorption of monolayer graphene can reach $-4.076 \mathrm{~dB}$, which is 17 times of the absorption of monolayer graphene in air. From Eq. (1), it can be seen that only the working light wave is at $1550 \mathrm{~nm}$, and
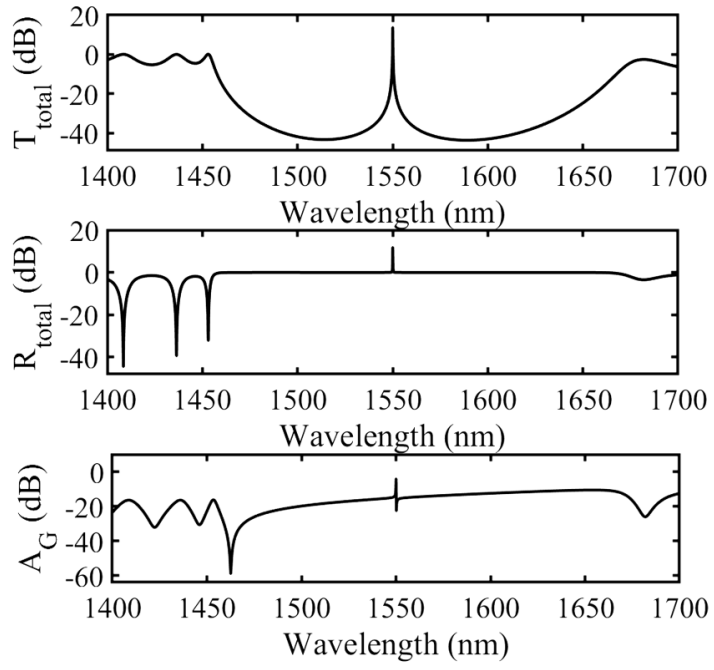

Fig. 2. Transmission and reflection spectra of the whole structure and the absorption spectra of the graphene layer.

the refractive index of the A and B layers can strictly satisfy the PT symmetry condition when $\omega_{0}=1550 \mathrm{~nm}$ is opted as the central wavelength (the real part of the gain-loss layer is even symmetric, and the imaginary part is odd symmetric). Under such circumstances, the PT symmetry structure has the strongest amplification effect on the transmission and reflection of the incident light, and the graphene-light interactions are tremendously enhanced; this is the dominating cause for the ultrastrong absorption of graphene. As can be seen from Eq. (8) and Fig. 2, both simulation results and theoretical analysis agree very well.

Macroscopic Lorentz oscillation intensity of the underlying PT symmetry photonic crystal, the incident light angle, and the chemical potential are the predominant factors affecting graphene absorption. The following analysis focuses on the influence of macroscopic Lorentz oscillation intensity, the incident light angle, and the chemical potential on graphene absorption.

The value of macroscopic Lorentz oscillation intensity $\alpha$ has a prodigiously greater influence on the imaginary part of the gainloss dielectric layer, and then it affects the resonance coupling effect of the whole structure. In order to demonstrate the effects of $\alpha$ on the absorption of graphene, firstly, keeping the other parameters constant, we present the graphene absorption
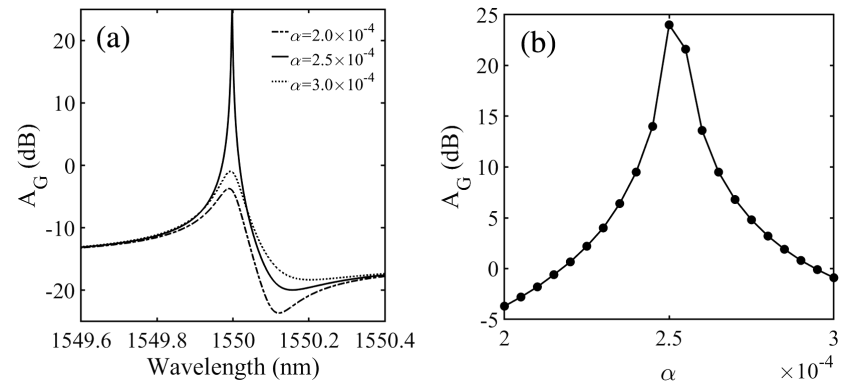

Fig. 3. (a) Absorption spectrum of graphene with different $\alpha$ values; (b) variation in the absorption at $1550 \mathrm{~nm}$ as a function of $\alpha$. 
spectrum with different $\alpha$ values in Fig. 3(a). The results show that the peak position of graphene absorption, with different $\alpha$ values, remains unchanged, while the absorption of graphene changes remarkably. Figure 3(b) presents the graphene absorption for $\alpha$ to be changed from $2 \times 10^{-4}$ to $3 \times 10^{-4}$ in steps of $0.1 \times 10^{-4}$, where the graphene absorption manifests a tendency to increase first and then decrease. The maximum absorption of monolayer graphene can reach $24.5 \mathrm{~dB}$ when $\alpha=2.5 \times 10^{-4}$, which is five orders of magnitude higher for monolayer graphene absorption than in air; this is because the resonant coupling effect of the structure is the strongest at $1550 \mathrm{~nm}$ when $\alpha=2.5 \times 10^{-4}$. The PT symmetry structure in the bottom layer has the maximum efficiency of converting pump energy into incident light wave electromagnetic energy, and the transmission and reflection of the structure are much higher than one, thus amplifying the absorption efficiency of the top layer of graphene. Notice that there is a small fluctuation in the value of $\alpha$ near the peak value, which will also have a great impact on the absorption of graphene. For practical application, it is a challenge to control $\alpha$ in this order of magnitude. Accordingly, we select $\alpha=2.5 \times 10^{-4}$ for the following calculation.

The influences of the incident light angle on graphene absorption and the absorption peak position are investigated, and the angle response $2 \mathrm{D}$ views of graphene absorption are plotted in Fig. 4. It is clearly seen that the absorption mode, deviating from on-resonance position, has a blue shift, and, simultaneously, the absorption drops from $24.5 \mathrm{~dB}$ to $1.3 \mathrm{~dB}$ quickly with the increasing incident light angle although the incident angle is changed only $10^{\circ}$.

Table 1 details the absorption peak position and the absorption peak value of graphene with different incident angles. The starting peak position of the absorption shifts from $1550 \mathrm{~nm}$ to $1541 \mathrm{~nm}$. Due to such angular susceptibility, care is taken to ensure that the graphene absorption is maximum; thus, the vertical incident light is still selected. The electro-modulation properties of graphene absorption based on the electro-optic effect of $\mathrm{LiNbO}_{3}$ crystal are discussed below.

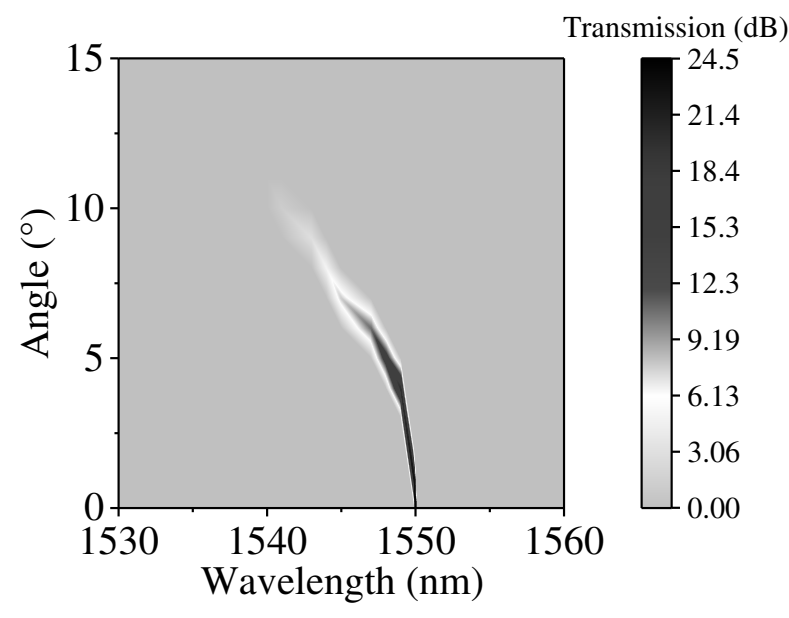

Fig. 4. Angle responses of graphene absorption.
Table 1. Absorption Peak Position and Peak Value at Different Incident Light Angles.

\begin{tabular}{|c|c|c|c|c|c|c|}
\hline Incident angle $\left({ }^{\circ}\right)$ & 0 & 2 & 4 & 6 & 8 & 10 \\
\hline Wavelength (nm) & 1550 & 1549.5 & 1549 & 1547 & 1544 & 1541 \\
\hline Absorption $(\mathrm{dB})$ & 24.5 & 22.4 & 19.9 & 10.7 & 5.2 & 1.3 \\
\hline
\end{tabular}
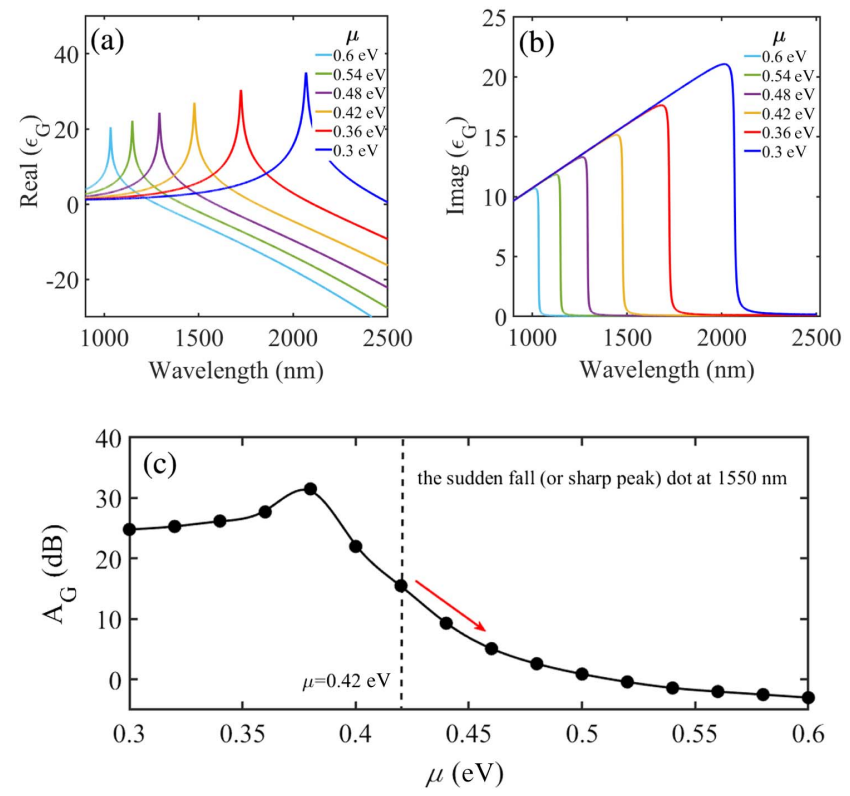

Fig. 5. (a) and (b) Real and imaginary parts of the complex permittivity of graphene for different chemical potential $\mu_{i}$ (c) the absorption of graphene as a function of the chemical potential $\mu$.

In Figs. 5(a) and 5(b), we give the real and imaginary parts of wavelength-dependent $\varepsilon_{\mathrm{G}}$, respectively, with the chemical potential $\mu$ to be changed from 0.3 to $0.6 \mathrm{eV}$ in steps of $0.06 \mathrm{eV}$. We can find in Fig. 5 that a sharp peak in the real part of $\varepsilon_{\mathrm{G}}$ and a sudden fall in the imaginary part of $\varepsilon_{\mathrm{G}}$ have a quick blue shift. It can be known from literature ${ }^{[25]}$ that when the wavelength is larger than the position of the sudden fall (or sharp peak) dot, the graphene absorption efficiency tends to be zero.

According to the sudden fall (or sharp peak) dot position, with the change of the chemical potential $\mu$, it is clearly seen in Fig. 5 that when the chemical potential is $\mu=0.42 \mathrm{eV}$, the sudden fall (or sharp peak) dot position is at $1550 \mathrm{~nm}$; that is, when the chemical potential exceeds $0.42 \mathrm{eV}$, the absorption of the $1550 \mathrm{~nm}$ light wave by monolayer graphene tends to zero. For the proposed structure, in Fig. 5(c), we calculated the absorption of graphene at different chemical potentials for the investigated wavelength of $1550 \mathrm{~nm}$. Apparently, the absorption of graphene begins to drop gradually when the chemical potential is close to or more than $0.42 \mathrm{eV}$. Accordingly, when setting the chemical potential parameter, the value should be less than $0.42 \mathrm{eV}$. According to the calculation results in Fig. 5(c), we take 
$\mu=0.38 \mathrm{eV}$ as the final optimization result in this paper. At this time, the absorption of monolayer graphene can reach $31.5 \mathrm{~dB}$, which is much higher than the corresponding graphene absorption $(-0.555$ to $0 \mathrm{~dB})$ in Refs. [9-11].

It can be straightforwardly seen from Eq. (3) that the refractive index of $\mathrm{LiNbO}_{3}$ crystal is related to the external electric field intensity, and the change of electric field intensity will lead to the change of the $\mathrm{LiNbO}_{3}$ crystal refractive index, so the position of the absorption peak will produce a red shift or blue shift. Accordingly, the absorption efficiency of graphene for the $1550 \mathrm{~nm}$ communication light wave will decrease.

In order to demonstrate that the absorption of graphene can be modulated largely to realize an electrically switched absorption effect, in Fig. 6(a), the electric field intensity variation range of the $y$ direction is selected from $-0.02 \mathrm{~V} / \mathrm{nm}$ to $0.02 \mathrm{~V} / \mathrm{nm}$, and the absorption values of graphene are calculated at different electric field values. It can be seen that even if the external electric field intensity only changes by $0.02 \mathrm{~V} / \mathrm{nm}$, it causes significant changes in graphene absorption. Therefore, the modulation of graphene absorption can be achieved by setting the value of the external electric field intensity. For the structure proposed in literature ${ }^{[26]}$, when realizing the unidirectional reversible properties of total reflection and total absorption, unidirectional reversible light modulation is required by switching the external electric field from $0.224 \mathrm{~V} / \mathrm{nm}$ to $-0.195 \mathrm{~V} / \mathrm{nm}$. Meanwhile, for the modulation of optical wave absorption in the narrow-band wave domain, the electric field needs to switch from $0.42 \mathrm{~V} / \mathrm{nm}$ to $-0.343 \mathrm{~V} / \mathrm{nm}$. The higher modulation threshold of reflection and absorption will be a challenge for practical application.

Modulation depth is a key indicator to describe the modulation capability of the structure, which is defined as
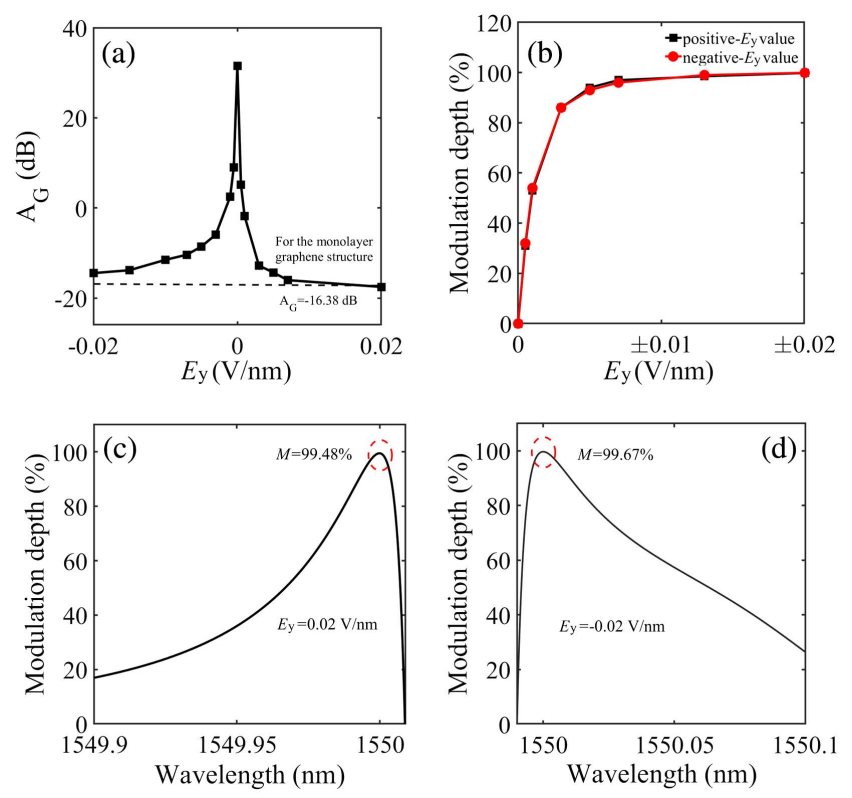

Fig. 6. (a) Absorption of graphene with different $E_{y}$ values; (b) modulation depth for different $E_{y}$ values; (c) and (d) modulation depth for $E_{y}= \pm 0.02$ $\mathrm{V} / \mathrm{nm}$.

$$
M=\frac{A_{0}-A}{A_{0}},
$$

where $A_{0}$ is the absorption of graphene for $E_{y}=0 \mathrm{~V} / \mathrm{nm}$, and $A$ is the corresponding absorption for the other values of $E_{y}$. Figure 6(b) shows the modulation depth of the structure under different electric field intensities; as the electric field intensity increases, the modulation depth of the structure increases gradually. When the electric field intensity is $\pm 0.02 \mathrm{~V} / \mathrm{nm}$, the modulation depth of graphene absorption reaches nearly 100\%, as exhibited in Figs. 6(c) and 6(d). Hence, this electrically switchable absorption of the structure makes it an excellent choice for the design of switchable absorbers or modulators of electromagnetic waves ${ }^{[27]}$.

In addition to modulation depth, the operation speed is also used to describe the structure performance of the graphene modulator, which can be estimated by the formula ${ }^{[28]}$

$$
f=\frac{1}{2 \pi R C},
$$

where $R$ is the ohmic resistance of graphene, and $C$ is the capacitance of the system, $C=\varepsilon_{0} \varepsilon_{\mathrm{A}} \mathrm{S} / d_{\mathrm{A}}$. For typical optical beam size, the square surface area of the device $S=50 \mu \mathrm{m} \times 50 \mu \mathrm{m}$ (for a typical optical beam size), so $C$ is about $0.087 \mathrm{pF} R=$ $d_{\mathrm{G}} /\left(S \cdot \sigma_{\mathrm{G}}\right)$ for the communication wavelength of $1550 \mathrm{~nm}$, and the chemical potential energy $\mu=0.3 \mathrm{eV}$, which is about $0.224 \mathrm{k} \Omega$. Then, we can calculate the operation speed $f$ to be about $8.171 \mathrm{GHz}$.

\section{Conclusions}

In this paper, we investigated the electrically switchable absorption characteristics of graphene prepared on top of a novel 1DPC with PT symmetry structure theoretically. Due to the exact matching use of the gain-loss dielectric layers, the maximum graphene absorption can reach $31.5 \mathrm{~dB}$ for the typical communication wavelength of $1550 \mathrm{~nm}$. Additionally, the results also show that based on the electric-optical effect of $\mathrm{LiNbO}_{3}$ the modulation depth of graphene can reach nearly $100 \%$ by setting the external electric field intensity to $\pm 0.02 \mathrm{~V} / \mathrm{nm}$. Meanwhile, the operation speed is close to $8.171 \mathrm{GHz}$. This novel structure proposed here may potentially have important applications in optoelectronic devices (e.g., photodetectors) and optical communication devices (e.g., optical switches and absorption modulators).

\section{Acknowledgement}

This work was supported by the National Natural Science Foundation of China (Nos. 61307050 and 61701271) and the Natural Science Foundation of Shandong Province (No. ZR2016AM27). 


\section{References}

1. J. C. Meyer, A. K. Geim, M. I. Katsnelson, K. S. Novoselov, T. J. Booth, and S. Roth, "The structure of suspended graphene sheets," Nature 446, 60 (2007).

2. N. Wang, W. H. Tian, H. S. Zhang, X. D. Yu, X. L. Yin, Y. G. Du, and D. L. Li, "An easily fabricated high performance Fabry-Perot optical fiber humidity sensor filled with graphene quantum dots," Sensors 21, 806 (2021).

3. B. Y. Liu, C. Y. You, C. Zhao, G. L. Shen, Y. W. Liu, Y. F. Li, H. Yan, and Y. Z. Zhang, "High responsivity and near-infrared photodetector based on

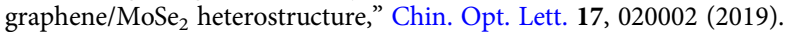

4. T. T. Liu, C. B. Zhou, and S. Y. Xiao, "Gain-assisted critical coupling for enhanced optical absorption in graphene," Nanotechnology 32, 205202 (2021).

5. D. D. Sun, M. Q. Wang, Y. Y. Huang, Y. X. Zhou, M. Qi, M. Jiang, and Z. Y. Ren, "Enhanced spatial terahertz modulation based on graphene metamaterial," Chin. Opt. Lett. 15, 051603 (2017).

6. Y. Z. Wang, Q. Wu, H. D. Wang, J. F. Liu, Z. Zheng, M. Zhang, and H. Zhang, "Thermally tunable microfiber knot resonator with flexible graphene heater," Chin. Opt. Lett. 19, 051301 (2021).

7. J. X. Huang, T. Fu, H. O. Li, Z. Y. Shou, and X. Gao, “A reconfigurable terahertz polarization converter based on metal-graphene hybrid metasurface," Chin. Opt. Lett. 18, 013102 (2020).

8. H. Y. Jiang, Y. Y. Tang, X. H. Zeng, R. W. Xiao, P. Lü, L. Wang, and Y. Q. Lu, "Visual measurement of the microscopic temperature of porous graphene based on cholesteric liquid crystal microcapsules," Chin. Opt. Lett. 18, 031201 (2020).

9. J. Gao, Y. L. Lan, and J. J. Wu, "Magnetically tunable multi-band absorption of graphene based on photonic crystal heterostructure," Chin. J. Lumin. 41, $624(2020)$.

10. G. Y. Zhu and Y. T. Fang, "Design of absorber at visible frequencies based on compound structure of one-dimensional photonic crystal and graphene," Chin. J. Lumin. 40, 1394 (2019).

11. G. S. Geng, X. L. Song, and D. S. Aereshji, “Tunable multiwavelength absorption in mid-IR region based on a hybrid patterned graphene-hBN structure," Opt. Express 27, 23576 (2019).

12. A. Safaei, S. Chandar, M. N. Leuenberger, and D. Chanda, "Wide angle dynamically tunable enhanced infrared absorption on large-area nanopatterned graphene," ACS Nano. 13, 421 (2019).

13. V. I. Klimov, A. A. Mikhailovsky, S. Xu, A. Malko, J. A. Hollingsworth, C. A. Leatherdale, H.-J. Eisler, and M. G. Bawendi, "Optical gain and stimulated emission in nanocrystal quantum dots," Science 290, 314 (2000).

14. N. Fakhroddin and A. Shahab, "PT-symmetric system based optical modulator,” Appl. Phy. B 124, 197 (2018).
15. D. Chatzidimitriou and E. E. Kriezis, "Optical switching through grapheneinduced exceptional points,” J. Opt. Soc. Am. B 35, 1525 (2018).

16. J. S. Cho, U. Paek, W. Han, and J. Heo, "Fabrication and heat treatment effects on absorption characteristics of glass fibers doped with PbTe semiconductor quantum dots," in Optical Fiber Communication Conference (2001).

17. A. A. Govyadinov, V. A. Podolskiy, and M. A. Noginov, "Active metamaterials: sign of refractive index and gain-assisted dispersion management," Appl. Phy. Lett. 91, 191103 (2007).

18. S. Ding and G. P. Wang, "Extraordinary reflection and transmission with direction dependent wavelength selectivity based on parity-time-symmetric multilayers," J. Appl. Phys. 117, 023104 (2015).

19. M. Minakshi and M. Sourangshu, "Analytical investigation to achieve the highest phase difference between two orthogonal components of light in lithium niobate based electro-optic system," Opt. Lett. 16, 338 (2020).

20. Y. Minet, H. Zappe, I. Breunig, and K. Buse, "Electro-optic control of lithium niobate bulk whispering gallery resonators: analysis of the distribution of externally applied electric fields," Crystals 11, 298 (2021).

21. X. S. Deng, M. Fang, X. G. Ren, Z. X. Huang, and X. L. Wu, "Ultra-sensitive bio-sensor based on trapped mode all-dielectric metasurface coating with graphene layer," Acta Photon. Sin. 48, 36 (2019).

22. J. Chen, S. Y. Chen, P. Gu, Z. D. Yan, C. J. Tang, Z. J. Xu, B. Liu, and Z. Q. Liu, "Electrically modulating and switching infrared absorption of monolayer graphene in metamaterials," Carbon 162, 187 (2020).

23. B. Liu, J. M. Shi, X. Y. Lv, Z. S. Chen, and Z. G. Li, "Application of photonic crystalline infrared stealth films on surface of vehicle engine compartments," Acta Photon. Sin. 50, 67 (2021).

24. J. T. Liu, N. H. Liu, J. Li, J. X. Li, and J. H. Huang, "Enhanced absorption of graphene with one-dimensional photonic crystal," Appl. Phy. Lett. 63, 402 (2012).

25. J. J. Wu and J. X. Cao, "Wideband absorption in one dimensional bilayergraphene embedded photonic multilayer structure," Superlattice. Microst. 140, 106437 (2020).

26. Y. T. Fang, Y. C. Zhang, and J. Xia, "Reversible unidirectional reflection and absorption of PT-symmetry structure under electro-optical modulation," Opt. Commun. 416, 25 (2018).

27. J. H. Shi, Z. J. Li, D. K. Sang, Y. J. Xiang, J. Q. Li, S. Zhang, and H. Zhang, “THz photonics in two dimensional materials and metamaterials: properties, devices and prospects," J. Mater. Chem. C 6, 1291 (2018).

28. S. Doukas, A. Chatzilari, A. Dagkli, A. Papagiannopoulos, and E. Lidorikisa, "Deep and fast free-space electro-absorption modulation in a mobility-independent graphene-loaded Bragg resonator," Appl. Phy. Lett. 113, 011102 (2018). 